\title{
Editorial
}

\section{New Developments in Mathematical Control and Information for Fuzzy Systems}

\author{
Hamid Reza Karimi, ${ }^{1}$ Mohammed Chadli, ${ }^{2}$ and Peng Shi ${ }^{3,4}$ \\ ${ }^{1}$ Department of Engineering, Faculty of Engineering and Science, University of Agder, 4898 Grimstad, Norway \\ ${ }^{2}$ Laboratory of Modeling, Information and Systems, University of Picardie Jules Verne, 80039 Amiens, France \\ ${ }^{3}$ College of Engineering and Science, Victoria University, Melbourne, VIC 8001, Australia \\ ${ }^{4}$ School of Electrical and Electronic Engineering, The University of Adelaide, Adelaide, SA 5005, Australia
}

Correspondence should be addressed to Hamid Reza Karimi; hamid.r.karimi@uia.no

Received 14 March 2013; Accepted 14 March 2013

Copyright (C) 2013 Hamid Reza Karimi et al. This is an open access article distributed under the Creative Commons Attribution License, which permits unrestricted use, distribution, and reproduction in any medium, provided the original work is properly cited.

\section{Discussion and Up-To-Date Overview}

Intelligence techniques, such as fuzzy logic approaches, have long been applied to dynamical systems with many important theoretical solutions and successful applications. The overall aim of this special issue is both to promote discussion among researchers actively working on applied fuzzy systems and to provide an up-to-date overview of the research directions and advance observer and controllers methods in the field. Of particular interest, the papers in this special issue are devoted to the development of mathematical methodologies analysis, control and observer problems of fuzzy systems, including nonlinear dynamics, fuzzy logic, and interdisciplinary topics with artificial intelligence. Potential topics include, but are not limited to (1) fuzzy controller design (robust control, adaptive control, and supervisory control), (2) fuzzy observer design (adaptive observers, sliding mode observers, and unknown inputs observers), (3) cascaded fuzzy systems and observers, (4) synchronization in fuzzy systems, (5) stochastic fuzzy systems, (6) robust fault detection and isolation, fault diagnosis, and fault-tolerant control of fuzzy systems, (7) applications of fuzzy controllers and observers to complex systems (including hardware and software development environments for fuzzy systems), (8) fuzzy logics, and (9) fuzzy modeling and optimization. For this special issue we solicit submissions from mathematicians, electri$\mathrm{cal} /$ control/mechanical engineers, and computer scientists. After a rigorous peer review process, 31 papers have been selected. These papers have covered both the theoretical and practical aspects of fuzzy systems in the broad areas of dynamical systems, mathematics, statistics, operational research, and engineering.

During the past decades, the problem of stability analysis of fuzzy systems has received significant attentions. In the paper entitled "Delay-dependent stability analysis of uncertain fuzzy systems with state and input delays under imperfect premise matching" by Z. Zhang et al., the robust stability and stabilization problems are studied for general nonlinear fuzzy systems with time-varying state and input delays. For obtaining a less conservative delay-dependent robust stability criterion, the authors introduce a novel augmented Lyapunov function with an additional triple-integral term to the stability analysis for the systems. Moreover, for improving the design flexibility and reducing the implementation cost of the fuzzy controller, they propose a new design approach different from the traditional PDC design technique, which means that the fuzzy model and the fuzzy controllers share different membership functions. Some less conservative stability conditions are obtained, and a new design approach is also proposed. The proposed stability conditions are less conservative in the sense of getting larger allowable time-delay and obtaining smaller feedback control gains, and the design flexibility is enhanced by arbitrarily selecting simple fuzzy membership functions. In the paper entitled "Stability analysis and stabilization of T-S fuzzy 
delta operator systems with time-varying delay via an inputoutput approach" by Z. Zhong et al., the stability analysis and stabilization problems are investigated for fuzzy delta operator systems with time-varying delay. The delta operator method is introduced to transform T-S fuzzy continuoustime systems into discrete-time systems, and the input-output (IO) approach is employed to deal with the stability analysis and control design of T-S fuzzy delta operator systems with time-varying delay. The main contribution of the paper is that the stability analysis and stabilization problems for fuzzy delta operator systems with time-varying delay are investigated by the IO Approach. A model approximation method is employed to transform the original system into an equivalent interconnected system, which is comprised of a forward subsystem with constant time-delays and a feedback one with delayed uncertainties, such that less conservative results are ensured.

The problems of modeling and identification have long been the main stream of research topics, and much effort has been made for fuzzy systems. In order to approximate any nonlinear systems, not just affine nonlinear systems, generalized T-S fuzzy systems where the control variables and the state variables are all premise variables are introduced in the paper entitled "A novel identification method for generalized T-S fuzzy systems" by L. Huang et al.. In order to improving the identification effect, a new method combined colony algorithm and genetic algorithm is proposed. Firstly, fuzzy spaces and rules are determined by using ant colony cluster algorithm to get the best one, and colony cluster algorithm has general optimizing capability, which is better than general cluster algorithm. Secondly, the state-space model parameters are optimized by genetic algorithm based on the least square method, which provided the better consequence parameters. In the paper entitled "Approximate analytic and numerical solutions to Lane-Emden equation via fuzzy modeling method" by D. Wang et al., a novel algorithm, called variable weight fuzzy marginal linearization (VWFML) method, is proposed to obtain the approximate analytic and numerical solutions to Lane-Emden equations. The main ideas of the VWFML method are as follows. Firstly, the authors need to transfer a group of data into fuzzy rules. Then, they, respectively, use two partition methods to divide the input universes and utilize fuzzy marginal linearization method to obtain the corresponding fuzzy system. By transferring initial value technology, the authors can solve these two fuzzy systems. Finally, they take weighted sum of these two solutions and obtain the approximate solutions of the Lane-Emden equations. When the Lane-Emden equation is unknown and only data information can obtained, many traditional numerical approximation methods could not solve it. The main contribution of this paper is that the authors can only use some data information to obtain the approximate analytic and numerical solutions to Lane-Emden equation with high accuracy. And this method is easy to be implemented and extended for solving other nonlinear differential equations. In the paper entitled "Bi-objective optimization method and application of mechanism design based on pigs' payoff game behavior" by L. Wang et al., a new bi-objective optimization game method is proposed. Two design goals can be regarded as two game players, the design variables set can be regarded as strategy subsets named $S_{1}$ and $S_{2}$, and the constraints in multiobjective problems can be regarded as constraints in the game method. Through the specific technological means, the design variables can be divided into each game players strategy subsets $\left(S_{1}\right.$ and $\left.S_{2}\right)$, and two payoff functions $u$ are constructed based on pigs' payoff game behavior. For optimization problems with preferred target, the designers need to emphasize one design goal. For this problem, there exist traditional methods such as weighting method (by adjusting the weight of each goal), hierarchical sequence method (by adjusting the objective optimization order), and goal programming method. In this paper, one new bi-objective optimization game method is proposed based on pigs' payoff game behavior for solving optimization problems with preferred target. It takes bi-objective optimization of luffing mechanism of compensative shave block; for example, the results show that the method can effectively solve the bi-objective optimization problems with preferred target (designers need to take the preferred target as the small pig side and take another target as the big pig side), the efficiency and accuracy are well, and the solution is obtained only through fewer game rounds. Although the Markowitz mean-variance (MV) portfolio optimization theory has been widely used, which leads the investment theory to a new era, in the paper entitled "Fuzzy investment portfolio selection models based on interval analysis approach" by $\mathrm{H}$. Guo et al., the authors found that this model has unintuitive problem in investment portfolio selection in economic decision. Thus this paper employs fuzzy set theory and extends it to a fuzzy investment portfolio selection model to solve this problem. Our model establishes intervals for expected returns and risk preference, which can take into account investors' different investment appetite and thus can find the optimal resolution for each interval. In the empirical part, we test this model in Chinese stocks investment and find that this model can fulfill different kinds of investors' objectives. Furthermore, investment risk can be decreased when we add investment limit to each stock in our model. The results indicate that fuzzy set theory is useful to avoid the problems of Markowitz mean-variance portfolio model and takes into account different expected return levels and risk preference levels. In the paper entitled "On interval valued supra fuzzy syntopogenous structure" by F. Sleim and H. Mustafa, the authors generalize the concept of supra fuzzy syntopogenous space by using the notion of interval valued set. Topology and its generalization proximity and syntopogenous are branches of mathematics which have many real life applications. They believe that the generalized topological structure suggested in this paper will be important base for modification of medical diagnosis, decision making, and knowledge discovery.

In the past decades, the issue of control design for fuzzy systems has received considerable research interests and has found successful applications in a variety of areas. An adaptive fuzzy sliding mode controller for a class of uncertain nonlinear systems is proposed in the paper entitled "Fuzzy sliding mode controller design using Takagi-Sugeno modelled nonlinear systems" by S. Bououden et al.. The unknown system dynamics and upper bounds of the minimum approximation 
errors are adaptively updated with stabilizing adaptive laws. The closed-loop system driven by the proposed controllers is shown to be stable with all the adaptation parameters being bounded. The performance and stability of the proposed control system is achieved analytically using the Lyapunov stability theory. In the paper entitled "Fuzzy variable structure control for uncertain systems with disturbance" by B. Wang et al., the authors focus on the fuzzy variable structure control for uncertain systems with disturbance. Specifically, the fuzzy control is introduced to estimate the control disturbance, and the switching control is included to compensate the approximation error and possess the characteristic of simpleness in design and effectiveness in attenuating the control chattering. In the paper entitled "Robust $H_{\infty}$ control for a class of uncertain switched fuzzy time-delay systems based on $T$-S models" by Y. Cui et al., the authors have developed a robust $H_{\infty}$ control with disturbance attenuation level $\gamma$ approach for a class of uncertain switched fuzzy time-delay systems based on T-S models. Each and every subsystem of the switched systems is an uncertain fuzzy one to which the PDC (parallel distributed compensation) controller of every subfuzzy system is proposed with its main condition given in a more solvable form of convex combinations. The closed-loop stability of the proposed robust $H_{\infty}$ control scheme is rigorously proved using Lyapunov theory. Finally, switching law of the state-dependent form achieving system quadratic stability of the switched fuzzy system is given. In the paper entitled "direct adaptive fuzzy sliding mode control with variable universe fuzzy switching term for a class of MIMO nonlinear systems" by G. Haigang et al., the authors developed a novel framework for the control of the MIMO nonlinear with model uncertainty and external disturbances. They also proposed a high-precision controller by the variable universe fuzzy control theory. In the paper entitled "A Fuzzy approach to robust control of stochastic nonaffine nonlinear systems" by T. Gang et al., the authors investigate the stabilization problem for a class of discrete-time stochastic nonaffine nonlinear systems (SNNS) based on generalized stochastic T-S fuzzy models. By using the function approximation capability of the stochastic T-S fuzzy models, the original SNNS can be exactly represented by a stochastic T-S fuzzy model with some norm bounded approximation errors as the uncertainty term on any compact set. In this way, the stabilization problem of the SNNS can be solved as a robust stabilization problem of the obtained uncertain stochastic T-S fuzzy models. By using a class of piecewise dynamic feedback fuzzy controllers and piecewise quadratic Lyapunov functions, robust semiglobally stabilization of SNNS can be formulated in terms of linear matrix inequalities. In the paper entitled "adaptive fuzzy tracking control for uncertain nonlinear time-delay systems with unknown dead-zone input" by C. Chiang, the problem of output tracking control is investigated for a class of uncertain nonlinear state timedelay systems containing unknown dead-zone input and unmatched uncertainties. In general systems, there exist some nonsmooth nonlinearities in the actuators, such as dead zone, saturation, and backlash. However, the deadzone characteristics in actuators may severely limit the performance of the systems. Also, time-delay characteristic and the existence of uncertain elements usually confronted in engineering systems may degrade the control performance and make the systems unstable. The main features of the proposed robust adaptive fuzzy controller are summarized as follows. (i) An adaptive law is used to estimate the properties of the dead-zone model intuitively and mathematically, without constructing a dead-zone inverse. (ii) Fuzzy logic systems with some appropriate learning laws are applied to approximate the nonlinear gain function and the upper bounded functions of uncertainties. (iii) The unknown upper bound of the uncertainties caused by approximation (or fuzzy modeling) error is estimated by a simple adaptive law. (iv) By means of Lyapunov stability theorem, the proposed controller cannot only guarantee the robust stability of the whole closed-loop system, but also obtain the good tracking performance. In this paper entitled "Fuzzy PD control of networked control systems based on CMAC neural network" by L. Huang and J. Chen, the main problem is how to design the fuzzy PD controller and combine with the cerebellar model articulation controller to compose a integral controller which used in the networked control systems effectively. In order to solve this problem, the switching control system between fuzzy PD and PD control is proposed. PD control in the small deviation is applied to obtain higher static control accuracy, and fuzzy PD control in large deviations is applied to obtain faster dynamic response and smaller overshoot. The paper entitled "Fuzzy control and connected region marking algorithm-based SEM nanomanipulation" by $\mathrm{D}$. Li et al. is motivated for manipulating the nanocomponent in SEM with telepresence as in macroscale. With the help of virtual reality and haptic technology, the SEM-based masterslave telenanomanipulation platform is established having the performance of security, reliable, and real time without force sensor. The CRM algorithm is introduced to process the 2D SEM image which provides effective position data of the objects for updating the virtual environment. The fuzzy control algorithm is adopted in the master-slave control to obtain relatively stable control variable to avoid damage of platform.

Over the past decades, the observer/filter problems of fuzzy systems have been investigated extensively, since they are very useful in signal processing and engineering applications. In the paper entiled "Robust observer design for Takagi-Sugeno fuzzy systems with mixed neutral and discrete delays and unknown inputs" by $\mathrm{H}$. Karimi and M. Chadli, a robust observer design is proposed for Takagi-Sugeno fuzzy neutral models with unknown inputs. The model consists of a mixed neutral and discrete delay, and the disturbances are imposed on both state and output signals. Delay-dependent sufficient conditions for the design of an unknown input T-S observer with time delays are given in terms of linear matrix inequalities. In the paper entitled "Observer-based robust adaptive fuzzy control for MIMO nonlinear uncertain systems with delayed output" by C. Chiang, the problem of controller design is considered for a class of MIMO nonlinear uncertain output-delay systems whose states are not available. The common feature of most previous results is the assumption that the controlled systems are free of uncertainties, or the uncertainties are assumed to be a bounded external 
disturbance. Therefore, the motivation of this paper is to synthesize an observer-based robust adaptive fuzzy control scheme to deal with the tracking control problem for a class of MIMO nonlinear uncertain systems with delayed output in the presence of uncertainties including the structural uncertainty. In the paper entitled "Unknown input observer design for fuzzy bilinear system: an LMI approach" by D. Saoudi et al., a new method to design a Fuzzy Bilinear Observer (FBO) with unknown inputs is developed for a class of nonlinear systems. The nonlinear system is modeled as a fuzzy bilinear model (FBM). This kind of T-S fuzzy model is especially suitable for a nonlinear system with a bilinear term. The proposed fuzzy bilinear observer subject to unknown inputs is developed to ensure the asymptotic convergence of the error dynamic using the Lyapunov method. An unknown input fuzzy bilinear fault diagnosis observer design is also proposed. Specifying for a class of nonlinear systems described by Takagi-Sugeno (T-S) model, in the paper, entitled "A reduced-order TS fuzzy observer scheme with application to actuator faults reconstruction" by D. Krokavec and A. Filasova, is newly defined the reducedorder T-S fuzzy observer, and it is demonstrated that the matching requirement, under which it can be disposed to actuator faults estimation, can be reflected in the observer design stipulation. The stability conditions, relying on the feasibility of an associated system of linear matrix inequalities (LMI), are derived and guarantee that the observer scheme asymptotically estimates actuator faults. In terms of fuzzy systems, the article provides a suitable new methodology and expands the theoretical basis of TS fuzzy model applications in control system design. The paper entitled "Terminal sliding mode control using adaptive fuzzy-neural observer" by D. Xu et al. proposed a dynamic approximation algorithm which is used to simplify the nonaffine nonlinear systems as affine nonlinear systems with time-varying parameters. And the time-varying parameters can be obtained by a filter. Next, an adaptive fuzzy-neural observer is designed to estimate the signals of position, velocity, and unknown functions. Terminal sliding mode control is used to design based on the observer. Stability analysis and simulations can show that the method is effective. The paper entitled "Filtering for discrete fuzzy stochastic time-delay systems with sensor saturation" by J. You et al. investigates the $H_{\infty}$ filtering problem for T-S fuzzy systems with time varying delay and sensor saturation. The communication channel between the plant and the filer is supposed to be imperfect, and random noise depending on the state and external disturbance is taken into account. The key method employed to handle with the time-varying delay is to develop the Scaled Small Gain (SSG) theorem to the stochastic systems. The main contribution is that this paper establishes a research approach that could handle with time varying delay and sensor saturation together, and both characteristics are always involved in many theoretical and practical problems. The paper entitled "Robust stabilization for continuous TakagiSugeno fuzzy system based on observer design" by Y. Manai and M. Benrejeb investigates the influence of new Parallel Distributed Controller (PDC) on the stabilization region of continuous Takagi-Sugeno (T-S) fuzzy models. Using a nonquadratic Lyapunov function, new sufficient stabilization criterion is established in terms of linear matrix inequality. The criterion examines the derivative membership function; an approach to determine state variables is given based on observer design.

The applications of various control schemes have received considerable research interests in the past decades. In the work entitled "An iterative procedure for optimizing the performance of the fuzzy-neural job cycle time estimation approach in a wafer fabrication factory" by T. Chen and Y. Wang, a classifying fuzzy-neural approach, based on the combination of principal component analysis (PCA), fuzzy cmeans (FCM), and back propagation network (BPN), is proposed to estimate the cycle time of a job in a wafer fabrication factory. The paper entitled "Switched two-level $H_{\infty}$ and robust fuzzy learning control of an overhead crane" by K. Hung et al. investigates the use of fuzzy techniques for modeling nonlinear plants as a combination of a nominal linear system and a T-S fuzzy blending of affine terms. This type of dynamic model significantly simplifies subsequent analysis and control designs, because assumptions on the plant dynamics can be significantly reduced. In the paper entitled " A two-wheeled self-balancing robot with the fuzzy PD control method" by J. Wu et al., the utility and effectiveness of soft computing approaches for a two-wheeled self-balancing robot with structured and unstructured uncertainties are presented. In this approach, precompensation of a hybrid fuzzy PD controller is proposed. The control scheme consists of a fuzzy logic-based precompensator followed by fuzzy PD control. Moreover, a fuzzy supervisory controller is used to supervise conventional proportional and derivative actions, such that the conventional gains are adapted online through fuzzy reasoning. Due to the influence of nonlinear friction, creep phenomenon occurs when the moving speed is low in the telescopic boom system of heavy-load transfer robot. To solve this problem and to improve control precision, $\mathrm{B}$. You et al. in the paper entitled "Low-speed control of heavyload transfer robot with long telescopic boom based on stribeck friction model" built a three-loop control nonlinear model of the system with the Stribeck friction disturbance model to simulate the motion of the telescopic boom in low speed. Fuzzy PID control was used to solve the problem of "flat-top" position tracking and "dead-zone" speed tracking. The creep phenomenon was eliminated, and the tracking accuracy and robustness of the system were also improved. The paper entitled "Using metaheuristic and fuzzy system for optimization of material-pull in a push pull flow logistics network" by A. Mehrsai et al. generally complies with a known problem in manufacturing environment, which is the coordination of heterogeneous material flows throughout supply chains and within every production plant. Alternative flows of material, following push and pull principles of control cause collection of inventory and WIP of materials as well as lags in delivery times. Model-based control of material flows regarding the interference of human in the entire process seems relatively very complex to practitioners in industries. In contrast, in this paper some material flow strategies are recommended to simplify and improve the flows throughout. Besides, some heuristics (i.e., genetic algorithm and simulated annealing) 
to solve the flow coordination are experimented here as well. Fuzzy logic as a suitable solution for solving human centered operations is reasonably explained and experimented in two applications, that is, control of pallets within an assembly system as well as normalization of multiobjective optimization problem. In the paper entitled "A novel evaluation model for hybrid power system based on vague set and dempster-shafer evidence theory" by D. Niu et al., due to advantages of vague set processing abundant uncertain and fuzzy information, it is chosen to determine basic decision matrix of evaluation model. Combining vague set with $\mathrm{D}-\mathrm{S}$ evidence theory, a novel evaluation algorithm is established and applied into the comprehensive benefit evaluation of hybrid power system. In the paper entitled " $H_{\infty}$ fuzzy control of DC-DC converters with input constraint," D. Saifia et al. study fuzzy control of DC-DC converters under actuator saturation. Because linear control design methods do not take into account the nonlinearity of the system, a T-S fuzzy models and a controller design approach are used. The input constraint is first transformed into a symmetric saturation which is represented by a polytopic model. Stabilization conditions for the $H_{\infty}$ state feedback system of DC-DC converters under actuator saturation are established using the Lyapunov approach. In the paper entitled " $A$ compound fuzzy disturbance observer based on sliding modes and its application on flight simulator," $\mathrm{Y}$. Wu et al. present a compound fuzzy disturbance observer based on sliding modes. The proposed method improves the performance of the disturbance inhibition when there exists huge modeling mismatch. Traditional methods use high-gain control, which may cause resonance in controlling elastic electromechanical systems, to inhibit equivalent disturbance. The proposed method employs fuzzy tools to deal with the primary part of the disturbance, and the residual disturbance is compensated by sliding mode control. The proposed method improves the robustness and performance of the system while avoiding the disadvantages of traditional methods. Finally, the paper entitled "Fuzzy formation control and collision avoidance for multiagent systems" by Y. Chang et al. investigates the formation control of leader-follower multiagent systems, where the problem of collision avoidance is considered. Based on the graph-theoretic concepts and locally distributed information, a neural fuzzy formation controller is designed with the capability of online learning. The learning rules of controller parameters can be derived from the gradient descent method. To avoid collisions between neighboring agents, a fuzzy separation controller is proposed such that the local minimum problem can be solved.

\section{Appendix}

\section{A. Accepted Papers According to Classified Topics}

\section{A.1. Papers on the Topic of Stability}

(1) Delay-dependent Stability Analysis of Uncertain Fuzzy Systems with State and Input Delays under Imperfect Premise Matching.
(2) Stability Analysis and Stabilization of T-S Fuzzy Delta Operator Systems with Time-Varying Delay via an Input-Output Approach.

\section{A.2. Papers on the Topic of Modeling/Identification}

(3) A Novel Identification Method for Generalized T-S Fuzzy Systems.

(4) Approximate analytic and numerical solutions to Lane-Emden equation via fuzzy modeling method.

(5) Bi-objective Optimization Method and Application of Mechanism Design Based on Pigs' Payoff Game Behavior.

(6) Fuzzy investment portfolio selection models based on interval analysis approach.

(7) On interval valued supra fuzzy syntopogenous structure.

\section{A.3. Papers on the Topic of Control for Fuzzy Systems}

(8) Fuzzy Sliding Mode Controller Design Using Takagi-Sugeno modelled Nonlinear Systems.

(9) Fuzzy variable structure control for uncertain systems with disturbance.

(10) Robust $H_{\infty}$ Control for A Class of Uncertain Switched Fuzzy Time-Delay Systems based on T-S Models.

(11) Direct Adaptive Fuzzy Sliding Mode Control with Variable Universe Fuzzy Switching Term for a class of MIMO Nonlinear Systems.

(12) A Fuzzy Approach to Robust Control of Stochastic Non-Affine Nonlinear Systems.

(13) Adaptive Fuzzy Tracking Control for Uncertain Nonlinear Time-Delay Systems with Unknown DeadZone Input.

(14) Fuzzy PD Control of Networked Control Systems Based on CMAC Neural Network.

(15) Fuzzy Control and Connected Region Marking Algorithm-based SEM Nanomanipulation.

\section{A.4. Papers on the Topic of Observer/Filter Design for Com- plex Systems}

(16) Robust Observer Design for Takagi-Sugeno Fuzzy Systems with Mixed Neutral and Discrete delays and Unknown Inputs.

(17) Observer-based Robust Adaptive Fuzzy Control for MIMO Nonlinear Uncertain Systems with Delayed Output.

(18) Unknown Input Observer Design for Fuzzy Bilinear System: an LMI Approach.

(19) A reduced-order TS fuzzy observer scheme with application to actuator faults reconstruction. 
(20) Terminal Sliding Mode Control using Adaptive Fuzzy-Neural Observer.

(21) Filtering for Discrete Fuzzy Stochastic TimeDelay Systems with Sensor Saturation.

(22) Robust Stabilization for Continuous TakagiSugeno Fuzzy System based on Observer Design.

\section{A.5. Papers on the Topic of Applications}

(23) An Iterative Procedure for Optimizing the Performance of the Fuzzy-neural Job Cycle Time Estimation Approach in a Wafer Fabrication Factory.

(24) Switched Two-Level $H_{\infty}$ and Robust Fuzzy Learning Control of an Overhead Crane.

(25) A Two-Wheeled Self-Balancing Robot with the Fuzzy PD Control Method.

(26) Low-Speed Control of Heavy-Load Transfer Robot with Long Telescopic Boom Based on Stribeck Friction Model.

(27) Using Meta-Heuristic and Fuzzy System for Optimization of Material-Pull In A Push-Pull Flow Logistics Network.

(28) A Novel Evaluation Model for Hybrid Power System Based on Vague Set and Dempster-Shafer Evidence Theory.

(29) $H_{\infty}$ Fuzzy Control of DC-DC Converters with Input Constraint.

(30) A Compound Fuzzy Disturbance Observer based on Sliding Modes and Its Application on Flight Simulator.

(31) Fuzzy Formation Control and Collision Avoidance for Multi-Agent Systems.

\section{Acknowledgments}

The guest editors would like to thank all the authors of this special issue for contributing the high quality papers, and we hope the reader will share our joy and find this special issue very useful. We are also very grateful to the reviewers for their efforts in reviewing the papers on time.

Hamid Reza Karimi Mohammed Chadli Peng Shi 


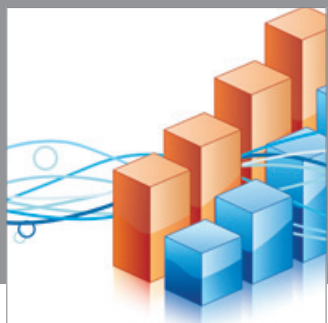

Advances in

Operations Research

mansans

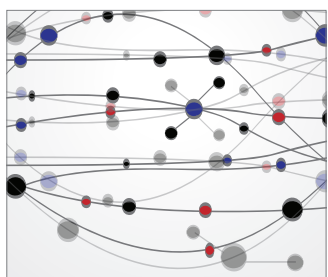

The Scientific World Journal
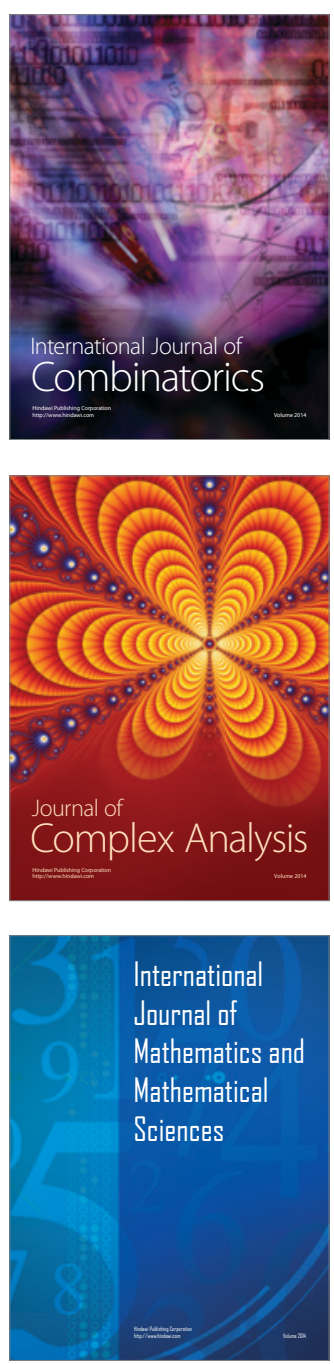
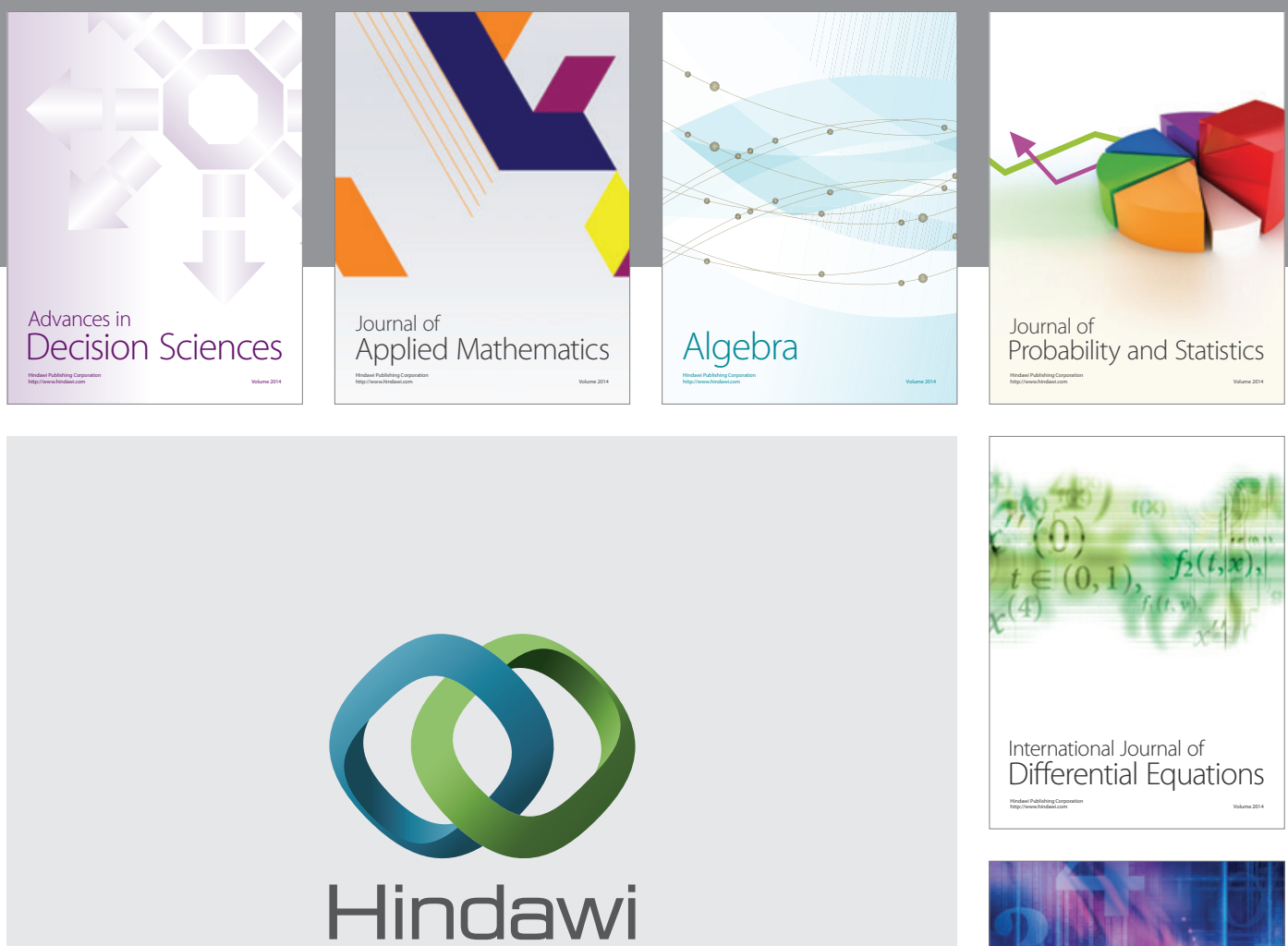

Submit your manuscripts at http://www.hindawi.com
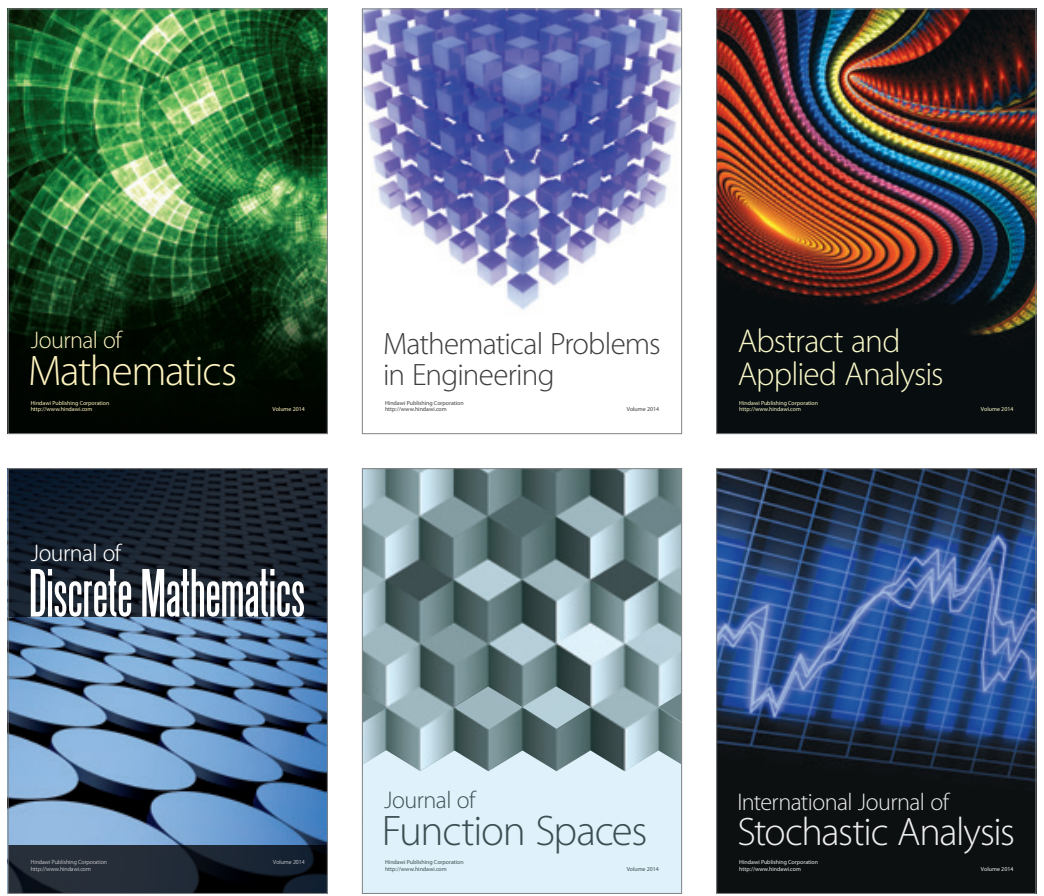

Journal of

Function Spaces

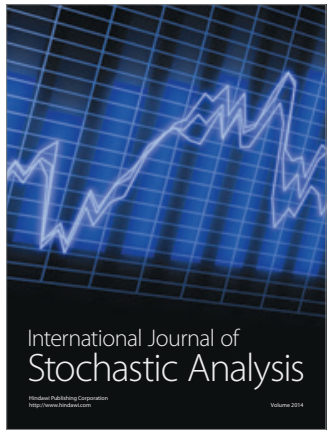

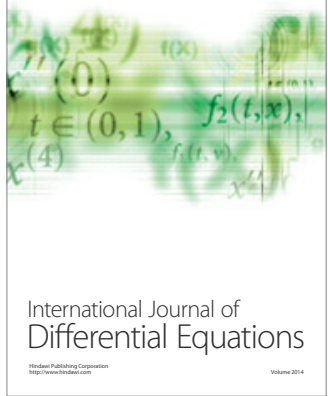
